\title{
A comparison of different diagnostic criteria of acute kidney injury in critically ill patients
}

Xuying Luo ${ }^{1 \dagger}$, Li Jiang ${ }^{1+}$, Bin Du², Ying Wen ${ }^{1}$, Meiping Wang ${ }^{1}$, Xiuming $\mathrm{Xi}^{{ }^{*}}$ and The Beijing Acute Kidney Injury Trial (BAKIT) workgroup

\begin{abstract}
Introduction: Recently, the Kidney Disease: Improving Global Outcomes (KDIGO) proposed a new definition and classification of acute kidney injury (AKI) on the basis of the RIFLE (Risk, Injury, Failure, Loss of kidney function, and End-stage renal failure) and AKIN (Acute Kidney Injury Network) criteria, but comparisons of the three criteria in critically ill patients are rare.
\end{abstract}

Methods: We prospectively analyzed a clinical database of 3,107 adult patients who were consecutively admitted to one of 30 intensive care units of 28 tertiary hospitals in Beijing from 1 March to 31 August 2012. AKI was defined by the RIFLE, AKIN, and KDIGO criteria. Receiver operating curves were used to compare the predictive ability for mortality, and logistic regression analysis was used for the calculation of odds ratios and $95 \%$ confidence intervals.

Results: The rates of incidence of AKI using the RIFLE, AKIN, and KDIGO criteria were $46.9 \%, 38.4 \%$, and 51\%, respectively. KDIGO identified more patients than did RIFLE (51\% versus $46.9 \%, P=0.001)$ and AKIN (51\% versus $38.4 \%, P<0.001)$. Compared with patients without AKI, in-hospital mortality was significantly higher for those diagnosed as AKI by using the RIFLE (27.8\% versus 7\%, $P<0.001)$, AKIN (32.2\% versus $7.1 \%, P<0.001)$, and KDIGO $(27.4 \%$ versus $5.6 \%, P<0.001)$ criteria, respectively. There was no difference in AKI-related mortality between RIFLE and $\mathrm{KDIGO}(27.8 \%$ versus $27.4 \%, P=0.815)$, but there was significant difference between AKIN and KDIGO (32.2\% versus $27.4 \%, P=0.006)$. The areas under the receiver operator characteristic curve for in-hospital mortality were $0.738(P<0.001)$ for RIFLE, $0.746(P<0.001)$ for AKIN, and $0.757(P<0.001)$ for KDIGO. KDIGO was more predictive than RIFLE for in-hospital mortality $(P<0.001)$, but there was no difference between KDIGO and AKIN $(P=0.12)$.

Conclusions: A higher incidence of AKI was diagnosed according to KDIGO criteria. Patients diagnosed as AKI had a significantly higher in-hospital mortality than non-AKI patients, no matter which criteria were used. Compared with the RIFLE criteria, KDIGO was more predictive for in-hospital mortality, but there was no significant difference between AKIN and KDIGO.

\section{Introduction}

Acute kidney injury (AKI) is very common, especially in the intensive care unit (ICU). It is also associated with increased mortality and a longer stay in the hospital [1-7]. There have been many definitions, such as acute renal failure and renal impairment, and this has made it difficult to compare results across studies. In 2004, the Acute Dialysis Quality Initiative group proposed a classification for AKI: the Risk, Injury, Failure, Loss of Kidney

\footnotetext{
* Correspondence: xxm2937@sina.com

${ }^{\dagger}$ Equal contributors

'Department of Critical Care Medicine, Fuxing Hospital, Capital Medical University, no. 20 Fuxingmenwai Street, Xicheng District, Beijing 100038, China

Full list of author information is available at the end of the article
}

Function, and End-stage Kidney Disease (RIFLE) classification, the first evidence-based consensus [8]. The classification includes three grades of severity of AKI (risk, injury, and failure) according to relative changes in serum creatinine $(\mathrm{SCr})$ and urine output and two outcomes (loss of kidney function and end-stage kidney disease, or ESKD). It has been evaluated in many studies of critically ill patients with AKI and has shown good relevance for diagnosing and classifying the severity of AKI as well as comparable predictive ability for mortality [7,9-13].

In 2007, the Acute Kidney Injury Network (AKIN) group proposed a modified version of the RIFLE classification, which aimed to improve the sensitivity of AKI criteria [14]. There were several changes: an absolute increase in $\mathrm{SCr}$ of 
at least $26.4 \mu \mathrm{mol} / \mathrm{L}$ was added to stage 1 ; patients starting RRT were classified as stage 3, irrespectively of SCr; and the change in glomerular filtration rate (GFR) and the two outcome classes were removed. AKI diagnosis was based on change between two creatinine values within a 48-hour period for AKIN classification and within a 1week window for RIFLE criteria. Severity of AKI in AKIN is staged over the course of 7 days by fold-change in creatinine from baseline.

The latest classification was proposed by the Kidney Disease: Improving Global Outcomes (KDIGO) Acute Kidney Injury Work Group, was based on the previous two classifications, and had the aim of unifying the definition of AKI [15]. According to this definition, AKI was diagnosed as an increase in SCr by at least $26.4 \mu \mathrm{mol} / \mathrm{L}$ within 48 hours or an increase in SCr to 1.5 times baseline, which is known or presumed to have occurred within 7 days before, or a urine volume of less than $0.5 \mathrm{~mL} / \mathrm{kg}$ per hour for 6 hours. For KDIGO criteria, the $26.4 \mu \mathrm{mol} / \mathrm{L}$ increase needs to be within 48 hours but a 1.5-fold increase can occur within 7 days to diagnose AKI; and the 1-week or 48-hour timeframe is for diagnosis of AKI, not for staging. A patient can be staged over the entire episode of AKI. Increase in SCr to 3 times baseline, or $\mathrm{SCr}$ of more than $4.0 \mathrm{mg} / \mathrm{dL}(354 \mu \mathrm{mol} / \mathrm{L})$, or starting RRT were all classified as stage 3 . KDIGO removes the $0.5 \mathrm{mg} / \mathrm{dL}$ increase for creatinine more than $4 \mathrm{mg} / \mathrm{dL}$ to diagnose stage 3. Besides, KDIGO explicitly states that a rolling baseline can be used over 48-hour and 7-day periods for diagnosis of AKI, but it is unclear how this is handled in RIFLE or AKIN. The definition and difference among the three criteria are shown in Additional file 1.

Many studies have compared RIFLE with AKIN in critically ill patients, but only a few have compared KDIGO with these criteria in critically ill patients with AKI. The purposes of this study were to determine the incidence of AKI in critically ill patients according to the RIFLE, AKIN, and KDIGO criteria and to compare their predictive ability.

\section{Materials and methods \\ Study cohort}

This study used a database from a prospective, multicenter, observational study which investigated the epidemiology of AKI in critically ill patients at 30 ICUs of 28 tertiary hospitals in Beijing, China, from 1 March to 31 August 2012. (For a complete list of those hospitals and the persons responsible for the acquisition of data, see Additional file 2.) All patients who were older than 18 years and who were consecutively admitted to any participating ICU during the observational period were enrolled. For patients with multiple admissions, only the first admission was considered. Patients who had ESKD, underwent any renal replacement therapy (RRT), received kidney transplantation during the past 3 months, or stayed in the ICU for less than 24 hours were excluded.

\section{Data collection}

Demographic data, dates of admission to the hospital and the ICU, primary diagnosis, co-morbidities, underlying chronic kidney disease, urine output (hourly or total urine volume in a 6-hour period), $\mathrm{SCr}$, the need for mechanical ventilation, and the use of vasoactive drugs were continuously recorded for 10 days or until discharge from the ICU, whichever occurred earlier. Dates of discharge from the ICU and the hospital were also documented. In-hospital mortality was recorded as the primary outcome. Non-renal Sequential Organ Failure Assessment (SOFA) scores [16], Acute Physiology and Chronic Health Evaluation (APACHE) II score, and related clinical data were also recorded.

\section{Definition of acute kidney injury}

The occurrence of AKI after ICU admission was determined by using the RIFLE, AKIN, and KDIGO criteria. Patients were categorized on the basis of $\mathrm{SCr}$ or urine output or both; the criteria that led to the worst classification were used. We did not use the GFR criteria. We used the lowest known SCr value during the past 3 months as the baseline creatinine in RIFLE and KDIGO criteria. For patients without known baseline, we used an estimated baseline or the lowest creatinine value during their stay in the ICU, whichever was lower. The baseline creatinine was estimated by using the simplified modification of diet in renal disease (MDRD) formula, assuming a GFR of $75 \mathrm{~mL} / \mathrm{min}$ per $1.73 \mathrm{~m}^{2}$, and customized for the Chinese population, assuming a GFR of $75 \mathrm{~mL} / \mathrm{min}$ per $1.73 \mathrm{~m}^{2}$ [17]. In this study, the baseline creatinine of 754 patients was not known; the MDRD formula was applied for 120 patients to estimate baseline creatinine; for 634 patients, the lowest creatinine values during stay in the ICU were used as baseline. For AKIN criteria, the ICU admission creatinine was used as the baseline, and a rolling baseline was also used over the course of 48 hours. Severity of AKI based on AKIN is staged over the course of 7 days by change in creatinine. For KDIGO criteria, the 1-week or 48-hour timeframe was for diagnosis of AKI, not staging; and a patient can be staged over the entire episode of AKI. Patients were evaluated daily by using the RIFLE, AKIN, and KDIGO criteria after admission, until day 10 or discharge from the ICU, and the maximum RIFLE, AKIN, and KDIGO within ICU hospitalization were recorded. The worst classification during the patient's ICU stay was used.

\section{Ethics}

The study was approved by the institutional review boards of Fuxing Hospital, Capital Medical University, and all other participating hospitals (Additional file 3). The institutional 
review board specifically approved the informed consent waiver because of the anonymous and purely observational nature of this study.

\section{Statistical analysis}

Data were analyzed by using SPSS 17.0.1 (SPSS Inc., Chicago, IL, USA). Non-normally distributed continuous variables were presented as median with interquartile range (IQR) and compared by Mann-Whitney $U$ test or Kruskal-Wallis analysis-of-variance test with Bonferroni correction. The categorical data were reported as proportions and compared by using the Fisher exact test. Logistic regression analysis was used to assess the association of each RIFLE, AKIN, and KDIGO category with in-hospital mortality. ICU patients without AKI were used as the reference group. The discriminative ability of the criteria to correctly predict mortality was assessed by calculating the area under the curve (AUC) of the receiver operating characteristic (ROC) curve. A comparison of the ROC curves was performed by using a method described by DeLong and colleagues [18]. A $P$ value of less than 0.05 was considered to be significant.

\section{Results}

During the study period, 9,049 patients were consecutively admitted to one of 30 ICUs. In total, 5,942 patients were excluded; of these patients, 110 were younger than
18 years old, one received renal transplantation during the past 3 months, and 95 patients had received RRT before admission to the ICU. A further 5,725 patients were excluded because their length of stay in the ICU was less than 24 hours, and 11 were excluded because of insufficient clinical recordings. Finally, 3,107 patients were enrolled. The characteristics of the whole cohort are shown in Table 1.

\section{Comparison of incidence of acute kidney injury}

AKI was diagnosed in 1,458 (46.9\%) patients by using the RIFLE classification: $20.8 \%$ with Risk, $12.4 \%$ with Injury, and $13.8 \%$ with Failure. According to AKIN criteria, AKI occurred in 1,193 (38.4\%) patients: $19 \%$ with stage $1,6.6 \%$ with stage 2 , and $12.8 \%$ with stage 3 . When KDIGO criteria were used, AKI occurred in 1,584 (51\%) patients: $23.1 \%$ with stage $1,11.8 \%$ with stage 2 , and $16 \%$ with stage 3 . The KDIGO criteria were more sensitive than RIFLE (51\% versus $46.9 \%, P<0.01)$ and AKIN (51\% versus $38.4 \%, P<0.001$ ).

A total of 259 patients received RRT within 10 days after ICU admission. According to the KDIGO and AKIN criteria, 247 of them were identified as AKI with stage 3; the other 12 patients without AKI received RRT for a number of reasons, including sepsis and drug overdose. On the basis of the RIFLE criteria, 245 patients were diagnosed with AKI: 14 with Risk, 33 with Injury, and 198 with Failure.

Table 1 Characteristics of patients at baseline

\begin{tabular}{|c|c|c|c|c|}
\hline Characteristics & No-AKI & AKI by KDIGO & AKI missed by RIFLE & AKI missed by AKIN \\
\hline Age, median (IQR) & $62(49-74)$ & $67(53-78)$ & $64(53-75)$ & $61(48-74)$ \\
\hline Male gender, n (\%) & $942(61.9)$ & $970(61.2)$ & $72(57.1)$ & $252(64.5)$ \\
\hline Surgical admission, n (\%) & $951(62.4)$ & $729(46)$ & $70(55.6)$ & $223(57)$ \\
\hline APACHE II score, median (IQR) & $12(8-16)$ & $17(12-23)$ & $15(11-22)$ & $15(10-21)$ \\
\hline SOFA score, median (IQR) & $4(2-7)$ & $6(3-10)$ & $6(3-9)$ & $6(4-10)$ \\
\hline Sepsis, n (\%) & $264(17.3)$ & $653(41.2)$ & $38(30.2)$ & $126(32.2)$ \\
\hline Mechanical ventilation, n (\%) & $957(62.8)$ & $1,132(71.5)$ & $101(80.2)$ & $275(70.3)$ \\
\hline Vasopressors, n (\%) & $466(30.6)$ & $864(54.5)$ & $67(53.2)$ & $201(51.4)$ \\
\hline Hypertension, n (\%) & $535(35.1)$ & $687(43.4)$ & $45(35.7)$ & $167(42.7)$ \\
\hline Diabetes, n (\%) & $212(13.9)$ & $320(20.2)$ & $23(18.3)$ & 74 (18.9) \\
\hline Chronic heart failure, n (\%) & $65(4.3)$ & $152(9.6)$ & $8(6.3)$ & $23(5.9)$ \\
\hline Chronic liver disease, n (\%) & $38(2.5)$ & $53(3.3)$ & $5(4)$ & $6(1.5)$ \\
\hline Chronic lung disease, n (\%) & $68(4.65)$ & $98(6.2)$ & $8(6.3)$ & $24(6.1)$ \\
\hline Chronic kidney diseases, n (\%) & $36(2.4)$ & $167(10.5)$ & $13(10.3)$ & $24(6.1)$ \\
\hline \multicolumn{5}{|l|}{ Baseline SCr } \\
\hline $2-3 \mathrm{mg} / \mathrm{dL}, \mathrm{n}(\%)$ & $5(0.3)$ & $30(1.9)$ & $4(3.2)$ & $3(0.8)$ \\
\hline 3-4 mg/dL, n (\%) & $1(0.1)$ & $18(1.1)$ & $2(1.6)$ & $1(0.3)$ \\
\hline$>4$ mg/dL, n (\%) & $3(0.2)$ & $27(1.7)$ & $2(1.6)$ & $4(1)$ \\
\hline
\end{tabular}

AKI, acute kidney injury; AKIN, Acute Kidney Injury Network; APACHE II, Acute Physiology and Chronic Health Evaluation II; IQR, interquartile range; KDIGO, Kidney Disease: Improving Global Outcomes; RIFLE, Risk, Injury, Failure, Loss of Kidney Function, and End-stage Kidney Disease; SCr, serum creatinine; SOFA, Sequential Organ Failure Assessment. 
The KDIGO criteria identified 126 more patients with AKI than the RIFLE criteria did: 106 with stage 1, 12 with stage 2, and 8 with stage 3 (Table 2). Among them, 124 patients were identified by an increase in creatinine alone, and the other two patients received RRT. Seventy patients were defined by KDIGO as stage 3 but not as failure by RIFLE (19 with Risk, 44 with Injury, and 8 without AKI), and 49 of them received RRT.

Compared with the AKIN criteria, KDIGO diagnosed 391 more patients as having AKI; 270 of them were categorized as stage 1, 84 as stage 2 , and 37 as stage 3 (Table 3 ). Among 391 patients, only 25 patients had chronic kidney disease. However, the median creatinine of these 391 patients on the first day of ICU admission was $118.6 \mu \mathrm{mol} / \mathrm{L}$ (IQR 78 to 159.7), which was much higher than the baseline: 118.6 (IQR 78 to 159.7) versus 70 (IQR 49 to 86), $P<0.001$.

\section{Comparison of outcomes In-hospital mortality}

Crude in-hospital mortality was significantly higher for AKI patients than for non-AKI patients, regardless of the definition used: the RIFLE (27.8\% versus 7\%, $P<0.0001)$, AKIN (32.2\% versus 7.1\%, $P<0.0001)$ and KDIGO $(27.4 \%$ versus $5.6 \%, P<0.0001)$ criteria. Mortality rate of patients identified as AKI by AKIN was higher than by KDIGO or RIFLE (32.2\% versus $27.4 \%, P=0.006$, and $32.2 \%$ versus $27.8 \%, P=0.013$; respectively) but did not differ significantly between RIFLE and KDIGO (27.8\% versus $27.4 \%$, $P=0.82$ ) (Table 4).

We also compared the in-hospital mortality of patients without AKI according different criteria and found that the patients identified by KDIGO but missed by AKIN or RIFLE had higher mortality than patients with noAKI based on KDIGO (12.8\% versus 5.6\%, $P<0.01$; $23 \%$ versus $5.6 \%, P<0.001)$.

The mortality rates of patients missed by the RIFLE criteria but identified by KDIGO as stage 1, stage 2, and stage 3 were $20.8 \%, 33.3 \%$, and $37.5 \%$, respectively. The mortality rates of those missed by the AKIN criteria but identified by KDIGO as stage 1 , stage 2 , and stage 3 were $9.6 \%, 19 \%$, and $21.6 \%$, respectively.

\section{Length of intensive care unit stays (alive)}

In our study, length of ICU stay was longer in patients with AKI than in those without AKI, no matter which criteria were used: the RIFLE (5 [3-10] versus 3 [2-6], $P<0.001$ ), AKIN (5 [3-11] versus 3 [2-6], $P<0.001)$, and KDIGO (5 [3-10] versus 3 [2-6], $P<0.001$ ) criteria. For patients missed by RIFLE or AKIN but identified by KDIGO, length of ICU stay was also longer than that of patients with no-AKI based on KDIGO (5 [3-8] versus 3 [2-6], $P<0.01$; [3-10] versus 3 [2-6], $P<0.01$; respectively).

\section{Predictive ability for mortality}

Irrespectively of which definition was used, AKI was independently associated with in-hospital mortality even after adjustment for age, gender, diabetes, hypertension, chronic kidney disease, chronic heart failure, and SOFA score (without renal component) (Table 5).

For patients diagnosed as AKI by KDIGO but not by RIFLE, AKI was also an independent risk factor of in-hospital mortality (odds ratio (OR) 4.498, 95\% confidence interval (CI) 3.727 to $5.429, P<0.001$ ) even after adjustment for age, gender, diabetes, hypertension, chronic kidney disease, chronic heart failure, and SOFA score (without renal component). Similarly, for patients identified as AKI by KDGIO but not by AKIN, AKI was an independent risk factor for mortality (OR 1.963, 95\% CI 1.139 to $2.898, P<0.01$ ).

The area-under-ROC curves for in-hospital mortality for RIFLE, AKIN, and KDIGO criteria were $0.738(P<0.001)$, $0.746(P<0.001)$, and $0.757(P<0.001)$, respectively. Compared with the RIFLE criteria, KDIGO had greater predictive ability for in-hospital mortality $(P<0.001)$ (Figure 1 and Table 6). But there was no significant difference between AKIN and KDIGO $(P=0.38)$.

\section{Patients with known baseline}

For patients with known baseline $(n=2,353)$, the rates of incidence of AKI according to RIFLE, AKIN, and KDIGO were $45.5 \%$, 39\%, and 50.6\%, respectively. The KDIGO criteria were more sensitive than RIFLE (50.6\% versus $45.5 \%, P<0.01$ ) and AKIN (50.6\% versus 39\%, $P<0.001$ ). Compared with patients without AKI, in-hospital mortality was significantly higher for those diagnosed as AKI by

Table 2 Agreement between RIFLE and KDIGO classifications

\begin{tabular}{llccccc}
\hline Definition & & \multicolumn{3}{c}{ RIFLE } \\
\cline { 3 - 6 } & & No-AKI & Risk & Injury & Failure & Total \\
\hline KDIGO & No-AKI & $1,523(49)$ & 0 & 0 & 0 & 0 \\
& Stage 1 & $106(3.4)$ & $612(19.7)$ & 0 & $323(49)$ \\
& Stage 2 & $12(0.4)$ & $15(0.5)$ & $341(11)$ & 0 & $318(23.1)$ \\
& Stage 3 & $8(0.3)$ & $18(0.6)$ & $44(1.4)$ & $428(13.8)$ & $498(11.8)$ \\
& Total & $1,649(53.1)$ & $645(20.8)$ & $385(12.4)$ & $428(13.8)$ & $3,107(100)$ \\
& & &
\end{tabular}


Table 3 Agreement between AKIN and KDIGO classifications

\begin{tabular}{|c|c|c|c|c|c|c|}
\hline \multirow[t]{2}{*}{ Definition } & & \multicolumn{5}{|c|}{ AKIN } \\
\hline & & No-AKI & Stage 1 & Stage 2 & Stage 3 & Total \\
\hline \multirow[t]{5}{*}{ KDIGO } & No-AKI & $1,523(49)$ & 0 & 0 & 0 & $1,523(49)$ \\
\hline & Stage 1 & $270(8.7)$ & $448(14.4)$ & 0 & 0 & $718(23.1)$ \\
\hline & Stage 2 & $84(2.7)$ & 100 (3.2) & $184(5.9)$ & 0 & 368 (11.8) \\
\hline & Stage 3 & $37(1.2)$ & $43(1.4)$ & $21(0.7)$ & 397 (12.8) & $498(16)$ \\
\hline & Total & $1,914(61.6)$ & 591 (19) & 205 (6.6) & 397 (12.8) & $3,107(100)$ \\
\hline
\end{tabular}

AKI, acute kidney injury; AKIN, Acute Kidney Injury Network; KDIGO, Kidney Disease: Improving Global Outcomes.

the RIFLE (27.8\% versus $7.3 \%, P<0.001)$, AKIN $(31.7 \%$ versus $7 \%, P<0.001)$, and KDIGO $(27.4 \%$ versus $5.7 \%, P<$ $0.001)$ criteria. There was no difference in AKI-related mortality between RIFLE and KDIGO $(P=0.82)$, but there was significant difference between AKIN and KDIGO (31.7\% versus $27.4 \%, P=0.031$ ). These results were identical to that of the whole study cohort.

\section{Discussion}

Numerous studies have compared the RIFLE and AKIN criteria for AKI. However, the incidence of AKI still varied. Based on these two criteria, the KDIGO criteria were recently proposed in order to unify the definition of AKI. To date, only a few previous studies have compared the incidence and mortality of AKI in critically ill patients according to these three definitions [19-21]. This is the first, large, multicenter study to compare these three different criteria in critically ill patients with AKI in China.

The incidence of AKI according to the KDIGO criteria was higher than that defined by RIFLE and AKIN, even after we excluded patients without known baseline creatinine. It was similar to the results of a study comparing definitions of AKI in hospitalized individuals in Boston [20] but differed from a retrospective study of patients after cardiac surgery, which concluded that incidence and outcome of AKI according to the RIFLE, AKIN, and KDIGO classification were similar [19]. The study of hospitalized patients conducted by Fujii and colleagues in Japan concluded that the rates of incidence of AKI

Table 4 In-hospital mortality according to AKI stratified by the RIFLE, AKIN, and KDIGO classification schemes

\begin{tabular}{lccc}
\hline Category & RIFLE & AKIN & KDIGO \\
\hline None (\%) & $115(7)$ & $136(7.1)$ & $86(5.6)$ \\
Risk/Stage 1 (\%) & $102(15.8)$ & $126(21.3)$ & $111(15.5)$ \\
Injury/Stage 2 (\%) & $111(28.8)$ & $69(33.7)$ & $103(28)$ \\
Failure/Stage 3 (\%) & $192(44.9)$ & $189(47.6)$ & $220(44.2)$ \\
Any category (\%) & $405(27.8)$ & $384(32.2)$ & $434(27.4)$ \\
\hline
\end{tabular}

AKI, acute kidney injury; AKIN, Acute Kidney Injury Network; KDIGO, Kidney Disease: Improving Global Outcomes; RIFLE, Risk, Injury, Failure, Loss of Kidney Function, and End-stage Kidney Disease. according to RIFLE, AKIN, and KDIGO were 11\%, 4.8\%, and $11.6 \%$, respectively [21]. KDIGO classified 126 (4.1\%) more patients with AKI than RIFLE did, the majority of which were patients with stage 1 . An in-depth analysis of these patients found that the majority (124 patients) were identified by a small increase in creatinine alone but that the remaining two patients received RRT at the same time. Firstly, we found that for some patients there was a decrease in creatinine after admission to the ICU, followed by a relative increase; these patients could be identified by KDIGO and AKIN because a rolling in-hospital baseline was used for the 48-hour rise, but not by RIFLE. Secondly, patients who received RRT would be classified as stage 3 by KDIGO and AKIN, irrespectively of SCr, but not by RIFLE.

When compared with AKIN, KDIGO diagnosed AKI in an additional 391 patients, including 25 patients with chronic kidney disease; these patients were predominantly stage 1 , followed by stage 2 and stage 3 . The median

Table 5 Association of different acute kidney injury category with mortality by multivariable logistic regression models

\begin{tabular}{lcc}
\hline Criteria & Odds ratio $(\mathbf{9 5 \%}$ Cl) & $P$ value \\
\hline RIFLE & $1.96(1.46-2.64)$ & $<0.001$ \\
Risk & $3.48(2.55-4.75)$ & $<0.001$ \\
Injury & $6.95(5.19-9.30)$ & $<0.001$ \\
Failure & & $<0.001$ \\
AKIN & $2.62(1.99-3.45)$ & $<0.001$ \\
Stage 1 & $4.63(3.22-6.65)$ & $<0.001$ \\
Stage 2 & $7.75(5.82-10.32)$ & $<0.001$ \\
Stage 3 & & $<0.001$ \\
KDIGO & $2.38(1.75-3.23)$ & $<0.001$ \\
Stage 1 & $4.31(3.09-6.02)$ & \\
Stage 2 & $8.54(6.31-11.56)$ & \\
Stage 3 & &
\end{tabular}

The model is adjusted for age, gender, diabetes, hypertension, chronic kidney disease, chronic heart failure, and Sequential Organ Failure Assessment (SOFA) score (without renal component). AKI, acute kidney injury; AKIN, Acute Kidney Injury Network; Cl, confidence interval; KDIGO, Kidney Disease: Improving Global Outcomes; RIFLE, Risk, Injury, Failure, Loss of Kidney Function, and End-stage Kidney Disease. 


\section{ROC Curve}

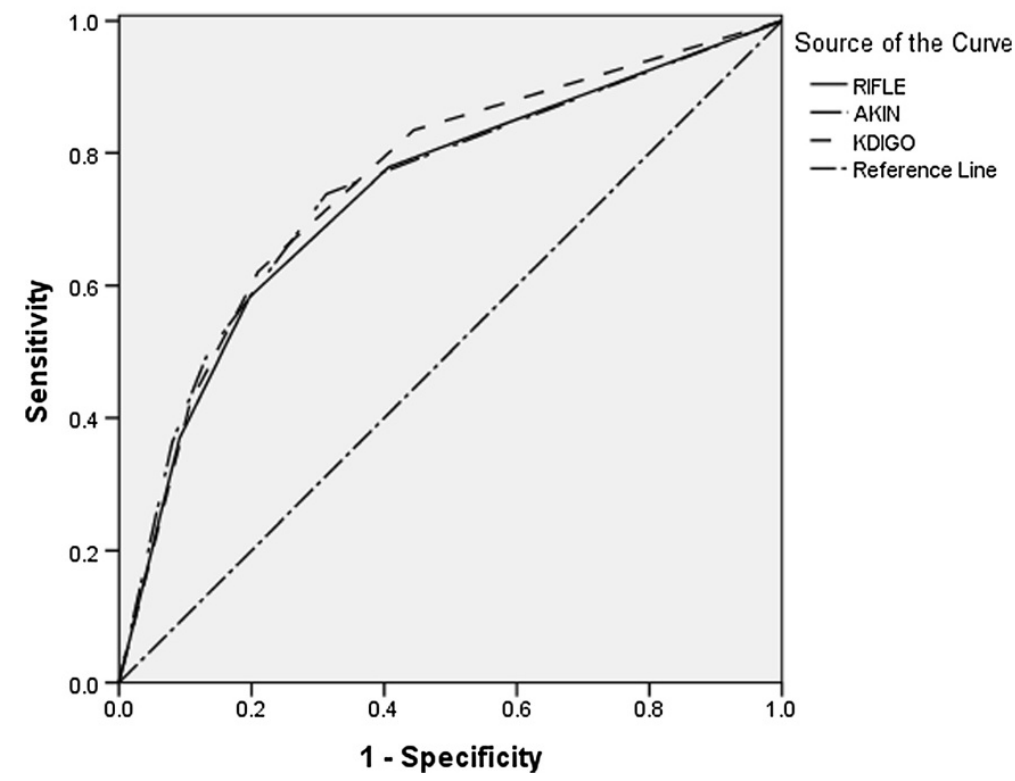

Figure 1 Area under the curves for RIFLE, AKIN, and KDIGO classification schemes comparing the predictive ability of RIFLE, AKIN, and KDIGO classification schemes for in-hospital mortality. AKIN, Acute Kidney Injury Network; KDIGO, Kidney Disease: Improving Global Outcomes; RIFLE, Risk, Injury, Failure, Loss of Kidney Function, and End-stage Kidney Disease; ROC, receiver operating characteristic. RIFLE: Area Under the Curve 0.738 ( $95 \% \mathrm{Cl} 0.713-0.762, \mathrm{P}<0.001$ ). AKIN: Area Under the Curve 0.746 (95\% Cl 0.721-0.770, P < 0.001). KDIGO: Area Under the Curve $0.757(95 \% \mathrm{Cl}$ $0.733-0.780, P<0.001$

creatinine level in these 391 patients on their first day of admission to the ICU was much higher than the baseline level, and this means that AKI may have been present on the day of ICU admission or even before. According to the AKIN criteria, AKI was diagnosed by two creatinine measurements within 48 hours. However, most patients did not have creatinine measured every day prior to the ICU admission: thus, when creatinine at ICU admission was used, some community-acquired AKI cases may have been missed [22-24]. In addition, patients with a slow reduction of renal function may have been missed by the
AKIN criteria [25]. The KDIGO definition reserved the baseline creatinine from RIFLE as well as a small increase in creatinine from AKIN criteria, allowing greater sensitivity than RIFLE and AKIN.

All definitions showed comparable and excellent associations with worse outcome according to increased severity of AKI. As for the predictive ability of these criteria, all were found to be significant predictors of increased mortality using multivariate analysis adjusting for age, gender, diabetes, hypertension, chronic kidney disease, chronic heart failure, and SOFA score. These findings were

Table 6 Predictive ability of RIFLE, AKIN, and KDIGO for in-hospital mortality

\begin{tabular}{|c|c|c|c|c|c|}
\hline Predictive factors & Cutoff point & Sensitivity, \% & Specificity, \% & +LR & $-\mathrm{LR}$ \\
\hline \multirow[t]{3}{*}{ RIFLE } & Risk-Failure & 77.9 & 59.3 & 1.91 & 0.37 \\
\hline & Injury-Failure ${ }^{a}$ & 58.3 & 80.3 & 2.96 & 0.52 \\
\hline & Failure & 36.9 & 90.0 & 4.05 & 0.69 \\
\hline \multirow[t]{3}{*}{ AKIN } & Stage 1 -Stage $3^{\mathrm{a}}$ & 73.9 & 68.7 & 2.36 & 0.38 \\
\hline & Stage 2-Stage 3 & 49.6 & 86.7 & 3.73 & 0.58 \\
\hline & Stage 3 & 36.4 & 92.0 & 4.52 & 0.69 \\
\hline \multirow[t]{3}{*}{ KDIGO } & Stage 1-Stage 3 & 83.5 & 55.6 & 1.88 & 0.30 \\
\hline & Stage 2-Stage $3^{\mathrm{a}}$ & 62.1 & 79.0 & 2.96 & 0.48 \\
\hline & Stage 3 & 42.3 & 89.3 & 3.94 & 0.65 \\
\hline
\end{tabular}

${ }^{2}$ Value is the best cutoff point. AKIN, Acute Kidney Injury Network; KDIGO, Kidney Disease: Improving Global Outcomes; + LR, positive likelihood ratio; $-L R$, negative likelihood ratio; RIFLE, Risk, Injury, Failure, Loss of Kidney Function, and End-stage Kidney Disease. 
identical to those of previous studies [3,4,26,27]. Patients missed by RIFLE but identified by KDIGO, most of which were classified as stage 1, had a longer length of ICU stay than no-AKI patients based on KDIGO. The patients diagnosed by KDIGO criteria as stage 1 but missed by RIFLE had much higher mortality than patients without AKI based on KDIGO (20.8\% versus $5.6 \%, P<0.001)$. Thus, we deduced that a small increase in creatinine might be accompanied by increased mortality. Similar results were observed in other studies [28,29]. A study by Wilson and colleagues determined that the magnitude of the decrease in creatinine generation rate may be correlated with the severity of illness [30]. In other words, the patients with a small increase in creatinine, accompanied by increased mortality and longer hospital stay, could be identified by KDIGO but not by RIFLE. The KDIGO definitions also showed a little better predictive ability than RIFLE did, according to the AUC curve for in-hospital mortality. For patients missed by AKIN but not by KDIGO, AKI was also an independent risk factor for mortality, but of low risk; and the mortality of these patients was only a little higher than that of no-AKI patients according to the KDIGO criteria $(12.8 \%$ versus $5.6 \%, P<0.01)$. In addition, the mortality of patients with AKI based on AKIN was a little higher than those on KDIGO (32.2\% versus $27.4 \%, P=0.006)$ and this was probably because KDIGO identified more patients in a mild severity level of AKI, with a relatively low mortality rate. According to the AUC curve, there was no significant difference between KDIGO and AKIN in the predictive ability for in-hospital mortality $(0.757$ versus $0.746, P=0.12$ ). Therefore, we concluded that KDIGO and AKIN were comparable on their predictive ability for in-hospital mortality. So whether this small increase in the mortality of these patients, identified by KDIGO but missed by AKIN, is of high risk requires more research. However, the study of hospitalized patients in Japan concluded that KDIGO and RIFLE achieved similar discrimination but that the discrimination of AKIN was inferior [21]. Given that their conclusion is different from ours, maybe more study is needed.

There are some limitations to our study. First, we used the simplified MDRD formula as baseline for patients without known baseline creatinine. In a prospective observational study, a good correlation of estimated as compared with observed baseline values was found for patients without chronic kidney disease [31]. Second, we did not have any records of creatinine during hospitalization but we did have records prior to ICU admission, and this may have caused the incidence of AKI by AKIN to be underestimated. The AKIN criteria recommend applying only the urine output criteria "following adequate fluid resuscitation", which is ambiguous. In our study, we did not adhere strictly to this recommendation. Third, we received hourly records of urine output for most patients, but for others only the total urine volume in a 6-hour period was recorded. A study by Etienne Macedo and colleagues [32] concluded that there was no significant difference between assessing urine output every hour or the total urine volume in a 6-hour period for the detection of episodes of oliguria, and the latter did not decrease their sensitivity for identifying patients with AKI. Finally, we did not have data regarding additional factors that could influence urine output, such as diuretic therapy.

\section{Conclusions}

The incidence of AKI in critically ill patients varied according to the criteria used. The KDIGO criteria identified more patients as AKI than RIFLE and AKIN did. Compared with the RIFLE criteria, KDIGO was more predictive for in-hospital mortality, but there was no significant difference between AKIN and KDIGO.

\section{Key messages}

- KDIGO identified more patients as AKI than RIFLE and AKIN did.

- AKI was independently associated with in-hospital mortality, irrespectively of which definition was used.

- For the patients diagnosed as AKI by KDIGO but not by RIFLE or AKIN, AKI was also an independent risk factor of mortality.

- KDIGO was more predictive for in-hospital mortality than RIFLE was.

\section{Additional files}

\begin{abstract}
Additional file 1: RIFLE, AKIN, and KDIGO criteria for AKI. The definition and difference among these three criteria are shown in detail. AKI, acute kidney injury; AKIN, Acute Kidney Injury Network; ESKD, end-stage kidney disease; GFR, glomerular filtration rate; KDIGO, Kidney Disease: Improving Global Outcomes; RIFLE, Risk, Injury, Failure, Loss of Kidney Function, and End-stage Kidney Disease; RRT, renal replacement therapy; Scr, serum creatinine.
\end{abstract}

Additional file 2: Members of the Beijing Acute Kidney Injury Trial (BAKIT) workgroup.

Additional file 3: All other ethical bodies that approved our study in the various centers involved.

\begin{abstract}
Abbreviations
AKI: acute kidney injury; AKIN: Acute Kidney Injury Network; AUC: area under the curve; Cl: confidence interval; ESKD: end-stage kidney disease;

GFR: glomerular filtration rate; ICU: intensive care unit; IQR: interquartile range; KDIGO: Kidney Disease: Improving Global Outcomes; MDRD: simplified modification of diet in renal disease; OR: odds ratio; RIFLE: Risk, Injury, Failure, Loss of Kidney Function, and End-stage Kidney Disease; ROC: receiver operating characteristic; RRT: renal replacement therapy; SCr: serum creatinine; SOFA: Sequential Organ Failure Assessment.
\end{abstract}

\section{Competing interests}

The authors declare that they have no competing interests. 


\section{Authors' contributions}

$X \mathrm{~L}$ and $\mathrm{LJ}$ designed and carried out the study, performed the statistical analysis, and drafted the manuscript. BD was involved in design and in acquisition of data and helped to revise the manuscript critically for important content. YW and MW were involved in the design and the statistical analysis. The Beijing Acute Kidney Injury Trial (BAKIT) Workgroup participated in acquisition and interpretation of data. XX conceived of the study, participated in its design, and helped to revise manuscript. All authors read and approved the final manuscript.

\section{Acknowledgments}

The study was supported by a grant from the Beijing Municipal Science \& Technology Commission, a government fund used to improve health-care quality (No. D101100050010058). It offered financial support for data collection.

\section{Author details}

${ }^{1}$ Department of Critical Care Medicine, Fuxing Hospital, Capital Medical University, no. 20 Fuxingmenwai Street, Xicheng District, Beijing 100038 China. ${ }^{2}$ Medical Intensive Care Unit, Peking Union Medical College Hospital, no. 1 Shuaifuyuan Wangfujing Dongcheng District, Beijing 100730, China.

Received: 26 February 2014 Accepted: 18 June 2014

Published: 8 July 2014

\section{References}

1. Chang $C H$, Lin CY, Tian YC, Jeng CC, Chang MY, Chen YC, Fang JT, Yang CW: Acute kidney injury classification: comparison of AKIN and RIFLE criteria. Shock 2010, 33:247-252.

2. Bagshaw SM, George C, Dinu I, Bellomo R: A multi-centre evaluation of the RIFLE criteria for early acute kidney injury in critically ill patients. Nephrol Dial Transplant 2008, 23:1203-1210.

3. Barrantes F, Tian J, Vazquez R, Amoateng-Adjepong Y, Manthous CA: Acute kidney injury criteria predict outcomes of critically ill patients. Crit Care Med 2008, 36:1397-1403

4. Nisula S, Kaukonen KM, Vaara ST, Korhonen AM, Poukkanen M, Karlsson S, Haapio M, Inkinen O, Parviainen I, Suojaranta-Ylinen R, Laurila JJ, Tenhunen J, Reinikainen M, Ala-Kokko T, Ruokonen E, Kuitunen A, Pettilä V, FINNAKI Study Group: Incidence, risk factors and 90-day mortality of patients with acute kidney injury in Finnish intensive care units: the FINNAKI study. Intensive Care Med 2013, 39:420-428.

5. Lopes JA, Fernandes P, Jorge S, Gonçalves S, Alvarez A, Costa e Silva Z, França C, Prata MM: Acute kidney injury in intensive care unit patients: a comparison between the RIFLE and the Acute Kidney Injury Network classifications. Crit Care 2008, 12:R110.

6. Zhou J, Yang L, Zhang K, Liu Y, Fu P: Risk factors for the prognosis of acute kidney injury under the Acute Kidney Injury Network definition: A retrospective, multicenter study in critically ill patients. Nephrology 2012, 17:330-337.

7. Wen $Y$, Jiang $L$, Xu Y, Qian CY, Li SS, Qin TH, Chen EZ, Lin JD, Ai YH, Wu DW, Wang YS, Sun RH, Hu ZJ, Cao XY, Zhou FC, He ZY, Zhou LH, An YZ, Kang $Y, M a X C, Y u X Y$, Zhao MY, Xi XM, DU B: Prevalence, risk factors, clinical course, and outcome of acute kidney injury in Chinese Intensive Care Units: a prospective cohort study. Chin Med J 2013, 126:4409-4416.

8. Bellomo R, Ronco C, Kellum JA, Mehta RL, Palevsky P, Acute Dialysis Quality Initiative workgroup: Acute Dialysis Quality Initiative workgroup: Acute renal failure - definition, outcome measures, animal models, fluid therapy and information technology needs: the Second International Consensus Conference of the Acute Dialysis Quality Initiative (ADQI) Group. Crit Care 2004, 8:R204-R212.

9. Hoste EA, Clermont G, Kersten A, Venkataraman R, Angus DC, De Bacquer D, Kellum JA: RIFLE criteria for acute kidney injury are associated with hospital mortality in critically ill patients: a cohort analysis. Crit Care 2006, 10:R73.

10. Lin CY, Chen YC, Tsai FC, Tian YC, Jenq CC, Fang JT, Yang CW: RIFLE classification is predictive of short-term prognosis in critically ill patients with acute renal failure supported by extracorporeal membrane oxygenation. Nephrol Dial Transplant 2006, 21:2867-2873.

11. Lopes JA, Jorge S, Neves FC, Caneira M, da Costa AG, Ferreira AC, Prata MM: An assessment of the rifle criteria for acute renal failure in severely burned patients. Nephrol Dial Transplant 2007, 22:285.

12. Uchino S, Bellomo R, Goldsmith D, Bates S, Ronco C: An assessment of the RIFLE criteria for acute renal failure in hospitalized patients. Crit Care Med 2006, 34:1913-1917.
13. Ostermann $M$, Chang RW: Acute kidney injury in the intensive care unit according to RIFLE. Crit Care Med 2007, 35:1837-1843.

14. Mehta RL, Kellum JA, Shah SV, Molitoris BA, Ronco C, Warnock DG, Levin A Acute Kidney Injury Network: report of an initiative to improve outcomes in acute kidney injury. Crit Care 2007, 11:R31.

15. Disease K: Improving Global Outcomes (KDIGO) Acute Kidney Injury Work Group: KDIGO Clinical Practice Guideline for Acute Kidney Injury. Kidney Inter Supp/ 2012, 2:1-138.

16. Vincent JL, Moreno R, Takala J, Willatts S, De Mendonça A, Bruining H, Reinhart CK, Suter PM, Thijs LG: The SOFA (Sepsis-related Organ Failure Assessment) score to describe organ dysfunction/failure, On behalf of the Working Group on Sepsis-Related Problems of the European Society of Intensive Care Medicine. Intensive Care Med 1996, 22:707-710.

17. Ma YC, Zuo L, Chen JH, Luo Q, Yu XQ, Li Y, Xu JS, Huang SM, Wang LN, Huang W, Wang $M, X u G B$, Wang HY: Modified glomerular filtration rate estimating equation for Chinese patients with chronic kidney disease. J Am Soc Nephrol 2006, 17:2937-2944.

18. DeLong ER, DeLong DM, Clarke-Pearson DL: Comparing the areas under two or more correlated receiver operating characteristic curves: a nonparametric approach. Biometrics 1988, 44:837-845.

19. Bastin AJ, Ostermann M, Slack AJ, Diller GP, Finney SJ, Evans TW: Acute kidney injury after cardiac surgery according to Risk/Injury/Failure/Loss/ End-stage, Acute Kidney Injury Network, and Kidney Disease: Improving Global Outcomes classifications. J Crit Care 2013, 28:389-396.

20. Zeng X, McMahon GM, Brunelli SM, Bates DW, Waikar SS: Incidence, Outcomes, and Comparisons across Definitions of AKI in Hospitalized Individuals. Clin J Am Soc Nephrol 2014, 9:12-20.

21. Fujii T, Uchino S, Takinami M, Bellomo R: Validation of the Kidney Disease Improving Global Outcomes Criteria for AKI and Comparison of Three Criteria in Hospitalized Patients. Clin J Am Soc Nephrol 2014, in print.

22. Srisawat N, Kellum JA: Acute kidney injury: definition, epidemiology, and outcome. Curr Opin Crit Care 2011, 17:548-555.

23. Bagshaw SM: Acute kidney injury: diagnosis and classification of AKI: AKIN or RIFLE? Nat Rev Nephrol 2010, 6:71-73.

24. Siew ED, Matheny ME, Ikizler TA, Lewis JB, Miller RA, Waitman LR, Go AS, Parikh CR, Peterson JF: Commonly used surrogates for baseline renal function affect the classification and prognosis of acute kidney injury. Kidney Int 2010, 77:536-542.

25. Ostermann M, Chang R, Riyadh ICU Program Users Group: Correlation between the AKI classification and outcome. Crit Care 2008, 12:R144.

26. Mandelbaum T, Scott DJ, Lee J, Mark RG, Malhotra A, Waikar SS, Howell MD, Talmor D: Outcome of Critically ill Patients with Acute Kidney Injury using the AKIN Criteria. Crit Care Med 2011, 39:2659-2664.

27. Yan X, Jia S, Meng X, Dong P, Jia M, Wan J, Hou X: Acute kidney injury in adult postcardiotomy patients with extracorporeal membrane oxygenation: evaluation of the RIFLE classification and the Acute Kidney Injury Network criteria. Eur J Cardiothorac Surg 2010, 37:334-338.

28. Chertow GM, Burdick E, Honour M, Bonventre JV, Bates DW: Acute kidney injury, mortality, length of stay, and costs in hospitalized patients. J Am Soc Nephrol 2005, 16:3365-3370.

29. Lassnigg A, Schmid ER, Hiesmayr M, Falk C, Druml W, Bauer P, Schmidlin D: Impact of minimal increases in serum creatinine on outcome in patients after cardiothoracic surgery: Do we have to revise current definitions of acute renal failure? Crit Care Med 2008, 36:1129-1137.

30. Wilson FP, Sheehan JM, Mariani LH, Berns JS: Creatinine generation is reduced in patients requiring continuous venovenous hemodialysis and independently predicts mortality. Nephrol Dial Transplant 2012, 27:4088-4494.

31. Bagshaw SM, Uchino S, Cruz D, Bellomo R, Morimatsu H, Morgera S, Schetz M, Tan I, Bouman C, Macedo E, Gibney N, Tolwani A, Oudemans-van Straaten HM, Ronco C, Kellum JA: A comparison of observed versus estimated baseline creatinine for determination of RIFLE class in patients with acute kidney injury. Nephrol Dial Transplant 2009, 24:2739-2744.

32. Macedo E, Malhotra R, Claure-Del Granado R, Fedullo P, Mehta RL: Defining urine output criterion for acute kidney injury in critically ill patients. Nephrol Dial Transplant 2011, 26:509-515.

\section{doi:10.1186/cc13977}

Cite this article as: Luo et al:: A comparison of different diagnostic criteria of acute kidney injury in critically ill patients. Critical Care 2014 18:R144. 